Revue

de Sémantique

et Pragmatique
Revue de Sémantique et Pragmatique

40 | 2016

Exclamation et intersubjectivité

\title{
Exclamation et intensité intersubjective dans un forum d'apprentissage à distance
}

Joséphine Remon

\section{(2) OpenEdition \\ 12 Journals}

Édition électronique

URL : http://journals.openedition.org/rsp/299

DOI : $10.4000 /$ rsp.299

ISSN : 2610-4377

Éditeur

Presses universitaires d'Orléans

Édition imprimée

Date de publication : 1 mars 2017

Pagination : 99-124

ISSN : 1285-4093

\section{Référence électronique}

Joséphine Remon, «Exclamation et intensité intersubjective dans un forum d'apprentissage à

distance ", Revue de Sémantique et Pragmatique [En ligne], 40 | 2016, mis en ligne le 01 mars 2018, consulté le 01 mai 2019. URL : http://journals.openedition.org/rsp/299 ; DOI : 10.4000/rsp.299 


\section{EXCLAMATION ET INTENSITÉ INTERSUBJECTIVE DANS UN FORUM D'APPRENTISSAGE À DISTANCE}

Joséphine Remon

Université Lyon 2, Laboratoire ICAR

\section{INTRODUCTION}

Le présent article se propose de contribuer à analyser l'exclamation du point de vue des relations intersubjectives qu'elle peut construire. Nous revisitons l'exclamation à la lumière des cadres théoriques de la linguistique de l'énonciation, de la Communication Médiatisée par les Technologies (CMT) et de l'Apprentissage des Langues Médiatisé par les Technologies (AMLT) (Guichon, 2012), dans le cadre particulier d'un travail collaboratif en anglais sur un forum d'apprentissage à distance. Nous ne considérons pas l'exclamation syntaxique mais les points d'exclamation, sur un corpus de productions écrites sur ce forum. Les points d'exclamation sont étudiés à la lumière de la notion de qualité de la relation à l'autre dans la CMT et à la lumière du contrat d'association sur lequel fonctionne un forum d'apprentissage, la ponctuation expressive jouant un rôle particulier dans les forums de discussion et nous éclairant en retour sur la dimension intersubjective des énoncés présentant un point d'exclamation. Nous présentons le contexte théorique de l'étude, puis le contexte pédagogique et le corpus et enfin les analyses.

\section{CONTEXTE THÉORIQUE}

Les outils théoriques qui nous permettent d'apporter un éclairage sur l'exclamation du point de vue de l'intersubjectivité proviennent de la linguistique de l'énonciation (Benveniste, 1974; Culioli, 1999) et de la pragmatique de la communication médiatisée (Herring, 2013). Les “traces de l'hétérogénéité 
énonciative et les phénomènes de modalisation" sont exploitables en effet dans la perspective de l'analyse du discours médiatisé par ordinateur, c'est-à-dire l'étude des manifestations pragmatico-linguistiques propres à la communication médiatisée (Devilla, 2006; Torres 2001). Kerbrat-Orecchioni (2011: 195) note à propos de la communication en ligne (synchrone) par opposition à la communication en présentiel que "ces échanges en ligne constituent bien, non un genre interactionnel particulier, mais une espèce particulière au sein de la grande famille de ce que l'on appelle des conversations".

\subsection{INTERSUBJECTIVITÉ ET ÉNONCIATION}

Certains auteurs mettent en évidence des éléments allant dans le sens du caractère transindividuel de l'acte de langage, notamment au sein de la théorie de l'énonciation. L'énonciation, telle que définie par Paillard (2013: 157) est «l'ensemble des déterminations (dont les formes qui la constituent sont la trace) qui interviennent dans la production de l'énoncé ». Dans ce cadre on recherche « des procédés linguistiques (...) par lesquels le locuteur imprime sa marque à l'énoncé, s'inscrit dans le message (implicitement ou explicitement) et se situe par rapport à lui (...) » (Kerbrat-Orecchioni, 1980: 32) ou « les marques linguistiques qui révèlent l'attitude du sujet parlant à l'égard de son interlocuteur, de lui-même et de son propre énoncé » (Devilla, 2006, p. 15). Filippi-Deswelle (2013 : 89) cite Culioli selon lequel (1999 : 45) on ne doit pas omettre « le mouvement qui est au cœur de tout acte de langage, cet ajustement des systèmes de repérage entre énonciateurs ». Il s'agit d'une " dynamique de régulation symbolique et interprétative, qui se rencontre aussi bien au plan transindividuel qu' au plan individuel » (Filippi-Deswelle, $2013: 89$ ). Culioli vise à « théoriser la relation intersubjective » (ibid. : 89-90). Comme l'explique Filippi-Deswelle (2013 : 93) « ce qui est privilégié [chez Culioli] comme objet de recherche, c'est que [1]e sujet est habité intérieurement par une activité de langage incessante qui peut, le cas échéant, se manifester extérieurement à travers les énoncés [...] produits en vue d'être interprétés par un autre sujet (...) ». L'interlocuteur est bien un co-énonciateur prenant part à une construction commune. C'est bien dans ce sens que les apports de Culioli peuvent éclairer une analyse de l'exclamation en lien avec l'intersubjectivité.

Filippi-Deswelle (2013 : 91) rappelle aussi que Benveniste se propose d'étudier « la subjectivité dans le langage » et « la communication intersubjective ». Là encore, l'intersubjectivité est fondatrice. Le sujet « se déclare locuteur et assume la langue, il implante l'autre en face de lui, quel que soit le degré de présence qu'il attribue à cet autre. Toute énonciation est, explicite ou implicite, une allocution, elle postule un allocutaire » (Benveniste, $1974: 82$ cité par Filippi-Deswelle, 2013 : 97 ). Jacques Bres (2013 : 9) va dans le même sens lorsqu'il trouve chez Benveniste (1970) et Bakhtine (1952) une «place 
structurante accordée à l'interaction verbale ». « Bakhtine pose, complémentairement à la dimension horizontale interlocutive de l'interaction (locuteur-interlocuteur), sa dimension verticale interdiscursive qui fait de chaque «énoncé » une réponse. » Cette vision est particulièrement pertinente, nous le verrons dans le cas d'un forum de discussion qui est au cœur de temporalités et spatialités multiples.

\subsection{INTERSUBJECTIVITÉ ET PRÉCONSTRUIT CONTRAIRE}

Mosès (2001:6) souligne que, chez Benveniste « la référence à la réalité (extérieure ou intérieure), doit être compris[e elle] aussi sur l'horizon de l'intersubjectivité, dans la mesure où l'identification de la portion de réalité à laquelle l'énonciation renvoie doit faire l'objet d'un accord entre les deux locuteurs ». Henning (2013 : 139) note qu'

«il est relativement rare que l'allocutaire laisse des traces dans la forme linguistique. En revanche, ses images apparaissent souvent au niveau de l'énoncé comme valeur par défaut des variables dont la forme linguistique ne donne aucune instruction quant à leur saturation. Ainsi, si l'on dit à quelqu'un « Ce mur n'est pas blanc », l'interlocuteur a tendance à penser que c'est lui qui, selon le locuteur, est source du point de vue positif. »

Ces énoncés avec sous-entendus "véhiculent une sorte de dialogue cristallisé » (2013 : 134) au sein d'une «polyphonie» (Angermuller, 2014). Pour donner un autre exemple, un marqueur comme « puisque » présente le contenu de la subordonnée comme pris en charge par l'allocutaire (ibid. : 136). Cette notion de prise en charge par l'allocutaire peut nous éclairer dans l'étude de l'exclamation du point de vue de l'intersubjectivité. Ainsi, Guillaume et Baumer (2014 : 11), à propos de l'exclamation en anglais, définissent de la manière suivante le « contexte exclamatif »: «le préconstruit contraire, c'est-à-dire l'attitude et les paroles du co-énonciateur, met en défaut les attentes de l'énonciateur, et l'oblige dès lors à hyper asserter la description de ce qui correspond à ses attentes ». Ces auteurs (ibid. : 4) expliquent : «[d]ans la mesure où la prise en compte de la dimension intersubjective dans l'interprétation exclamative nous semble essentielle, nous avons recours dans notre étude aux outils de la Théorie des Opérations Enonciatives d'A. Culioli, un cadre théorique qui donne toute sa place à la relation intersubjective ». Un marqueur discursif est « un mot ou une locution qui introduit une détermination spécifique portant sur telle ou telle composante de la scène énonciative » (Paillard, $2013:$ 160), les marqueurs discursifs intersubjectifs, quant à eux, notamment les interjections, « [actualisant] telle ou telle position dans l'espace intersubjectif » (ibid.: 166). On retrouve des opérations similaires avec la modalité, qui peut dans certains cas s'inscrire « dans un dialogue implicite avec ce que l'énonciateur suppose que son co-énonciateur pense » (Bouscaren et Chuquet, 1987 : 37, cité par Douay, 2003 : 13). A propos 
d'un marqueur comme though, par exemple, Filippi-Deswelle (2010) remarque qu'il « permet d'exhiber des dissociations énonciatives qui font place à la pensée de l'autre, que cet autre soit autrui à proprement parler (dissociation intersubjective), ou qu'il soit une autre part de soi-même (dissociation intrasubjective) » (cité par Mellet, 2011 : 4). La notion de monde contrefactuel ou hétérounivers (Martin, 1987) est éclairante à cet égard, c'est-à-dire l'univers de croyance d'un locuteur tel qu' « imaginé par un autre » (Romero, 2004 :7).

\subsection{INTERLOCUTIF / INTERPERSONNEL}

Douay (2003) analyse le chevauchement entre les notions de subjectivité et de modalité et fait le lien entre modalité et intersubjectivité. Son projet est de théoriser « la distinction entre relation interlocutive et relation interpersonnelle » (ibid. : 17) dans les modalités, la première étant sur le «terrain linguistique », la seconde étant sur le «terrain psychosocial » (ibid. : 5) : «De façon générale, le terme de «relation intersubjective» souffre de ce type d'ambiguïté chez la plupart des linguistes, qui l'appréhendent tantôt sur le terrain strictement linguistique de l'interlocution, tantôt sur le terrain psychosocial des relations interpersonnelles » (ibid. : 15).

Cette opposition entre interlocution et relations interpersonnelles nous semble pertinente en ce qui concerne l'étude de l'exclamation du point de vue de l'intersubjectivité, pour éventuellement même la dépasser et proposer une approche intégrative de l'interlocutif et de l'interpersonnel. Si l'intersubjectivité est au cœur de l'énonciation, les relations interpersonnelles sont « un facteur prédominant dans les biographies langagières » des apprenants de langue (Müller, 2016). Ainsi, sur un forum d'apprentissage de la langue à distance, l'intersubjectivité peut être comprise à plusieurs niveaux, comme constitutive de l'énonciation et mais aussi de l'apprentissage. Nous proposons donc de replacer l'exclamation, dans ses aspects intersubjectifs, dans le contexte complexe composé de l'interlocution, des relations interpersonnelles en synchronie (dans l'immédiateté du forum) et en diachronie également (dans le contexte d'autres échanges que peuvent avoir les participants ailleurs et par d'autres canaux de communication), et, dans le cas des apprenants de langue, des relations entre les différents états de leur moi langagier. Le contexte didactique donne ainsi une coloration particulière à cette intersubjectivité. En effet, puisqu'il s'agit d'un apprentissage, une évolution du moi est impliquée. L'intersubjectivité peut dès lors se concevoir entre pairs, ou entre apprenants et enseignant, mais aussi entre divers états de ses propres représentations sur la langue et entre divers états langagiers. On pourrait alors la qualifier d'intrasubjectivité.

Filippi-Deswelle (2013 : p. 101) note que toute énonciation est insérée « dans un champ de forces inter-sujets qui confère aux propos tenus une valeur 
nécessairement ajustée, c'est-à-dire susceptible d'être contestée par autrui. » C'est bien à l'intérieur de ce champ de forces que nous proposons de replacer l'exclamation, ainsi que dans le contexte des efforts énonciatifs menés dans le discours numérique collaboratif en vue de la réalisation d'un objectif commun, dans le cas particulier d'un apprentissage de langue étrangère.

Notre apport sur ces aspects, au vu de l'analyse de notre corpus, est de proposer une approche à travers une gradation et un enchâssement des opérations énonciatives et socioaffectives.

\subsection{MARQUES DE RELIANCE}

Quintin et Masperi (2010) apportent un éclairage sur ces aspects intersubjectifs avec le concept de reliance, qui donne tout son sens au projet de Douay (2003 : 5) de « théoriser la place de l'interlocuteur en montrant que le système de la langue s'organise, non pas autour de la personne du locuteur, mais autour de la relation interlocutive elle-même ». Ce projet semble d'autant plus valable dans un contexte d'échanges écrits sur un forum à distance. Les marques de reliance traduisent en effet la création de lien entre les membres d'un groupe d'apprentissage engagé dans un travail commun (Quintin \& Masperi, 2010 : 19). La reliance se décline en liance et alliance. La liance recouvre les stratégies énonciatives permettant de " cultiver la qualité de la relation à l'autre, le message déposé par l'énonciateur révélant une sollicitude affectueuse et bienveillante vis-à-vis du ou des destinataires » (ibid.: 39), par exemple l'utilisation de diminutifs, révélant « l'effort énonciatif consenti par le locuteur pour soigner la qualité de sa relation à l'autre » (ibid. : 40). L'alliance recouvre « les procédures énonciatives de type collaboratif », tels des commissifs, qui visent à «établir et à conforter le contrat d'association l'alliance entre les co-actants en vue de la réalisation du travail commun » (ibid. : 21). Dans le même ordre d'idée, Develotte et Codreanu (2010: 9) appellent ces traces des marqueurs de construction du lien social et de familiarité.

Paveau (2013), à propos des échanges sur les Réseaux Sociaux Numériques cette fois, émet l'idée que « les RSN sont des réseaux, c'est-à-dire des espaces qui sont construits des relations qu'ils permettent : sur le plan langagier, tous les énoncés produits sur les RSN sont conversationnels car ils supposent une relation ». Même si un forum n'est pas assimilable à un RSN, on y trouve cette même relation inhérente.

Ainsi, la nature intersubjective de l'acte énonciatif se combine avec la nature conversationnelle des énoncés produits sur le forum, et nous permet de mettre en relief le rôle particulier du point d'exclamation au sein de ce dispositif. La volonté de rendre la relation plus intense se révèle sur le forum à travers ces marqueurs. Nous proposons donc une hypothèse qui, loin d'aller dans le 
sens d'une exclusion de l'interlocuteur, va au contraire vers une interprétation du point d'exclamation comme marqueur d' «intensité intersubjective».

\subsection{PONCTUATION EXPRESSIVE ET COMMUNICATION MÉDIATISÉE}

Parmi les marqueurs de construction du lien social, certains chercheurs s'attachent en effet plus particulièrement à la ponctuation expressive. Anis $(1994,1998)$ oppose déjà ponctuation expressive et ponctuation syntaxique à propos de l'écriture télématique. Von Münchov et Rakotoelina (2006) parlent de « convivialité énonciative» (cité par Devilla, 2008 : 8). Devilla (ibid.) note qu' " au sein des forums de discussion la ponctuation expressive représente une ressource pour exprimer l'émotion ». Pour Crystal (2001) également, étirement vocalique ou consonantique et ponctuation expressive font partie des caractéristiques du Netspeak. La ponctuation non-standard et l'émulation prosodique sont données comme des caractéristiques de discussions par e-mail par Herring (2013).

Les points d'exclamation sont donc en effet un des marqueurs utilisés dans les analyses de la conversation numérique. Ainsi Jeanneau et Ollivier (2009 : 8 ), pour « analyser les aspects socio-affectifs des interactions en ligne », observent les «marqueurs d'identité » et « les marques d'expression d'un sentiment, marques verbales et non verbales, sous forme essentiellement d'émoticônes et de points d'exclamation ». Yun (2009 : 218) également, relève les points d'exclamation dans des échanges à distance synchrones à base textuelle (clavardage), qui peuvent marquer par exemple « un accord parfait avec la question du tuteur ». Gélis (2013 : 35) nous offre une fenêtre sur l'usage du point d'exclamation par un tuteur sur un forum d'apprentissage à distance, où l'on voit bien que ces points d'exclamation sont destinés aux interlocuteurs, dans une stratégie pour «garder la main »: «Alors de temps en temps, je marque STOP en majuscule avec 25 points d'exclamation pour remplir toute la ligne et je réponds plus rien jusqu'à temps qu'ils arrêtent de clavarder entre eux. »

Polge-Loï (2013), sur une interface blog, recherche les traces d'intersubjectivité à partir d'un exemple où celle-ci se manifeste par un conflit, avec la rencontre de "représentations de valeurs axiologiques différentes » (ibid. : 2). Ces traces sont notamment des points d'exclamation : « Les marques graphiques et typographiques [...] exprimant de l'émotion et renforçant l'attaque sont les sept points d'exclamation [...] et les points de suspension qui suggèrent au lecteur l'existence d'une tonalité ironique implicite. Les autres marques jouent plutôt un rôle d'adoucisseur de l'attaque : les dix points d'exclamation qui suivent la formule de salutation et le « $\mathrm{u} »$ triplé de [bisouuu] » (ibid. : 3). Devilla (2008: 3) quant à lui, au sein de la plateforme d'apprentissage Galanet, s'attache à analyser « la dimension des positionnements, les modalités énoncia- 
tives, et en particulier les modalités d'adresse et la ponctuation expressive [et] la dimension socio-affective, mettant en évidence les indicateurs révélateurs d'un sentiment d'appartenance à la communauté de parole de Galanet ». Il compte les messages se terminant par un ou plusieurs points exclamatifs (op. cit.: 7): « Si l'on considère la répartition par phases, les points exclamatifs sont proportionnellement plus nombreux dans les phases du scénario pédagogique dont la nature incite à chercher le contact (phase I "briser la glace", IV "dossier de presse" et épilogue), confirmant ainsi leur valeur relationnelle». Zourou (2006 : 299) cite le message de deux tutrices et le qualifie « d'acte discursif désespéré » avec la « répétition des points d'exclamation comme signe d'un état motivationnel intense », pour formuler un « appel explicite de reprise de contact» ces « marques de modalisation » étant « propres à l'écrit conversationnel en ligne».

\subsection{COMMUNICATION MÉDIATISÉE EN LANGUE ÉTRANGÈRE DANS UN CONTEXTE D'APPRENTISSAGE}

Si la communication médiatisée est déjà une situation d'énonciation spécifique, la communication médiatisée en langue étrangère dans un contexte d'apprentissage l'est encore plus, surtout si cet apprentissage prend une forme collaborative.

Hors situation d'apprentissage, Murie étudie l'anglais des forums du point de vue syntaxique, lexical et stylistique. Elle précise (2016:31) que « l'implantation de la relation discursive entre le locuteur et son allocutaire présumé est essentielle, car la relation discursive est le seul type de relation possible entre les membres d'un forum ». A propos des points d'exclamation, elle en remarque la multiplication (ibid. : 141-142) :

Même si le point d'exclamation est utilisé individuellement à 321 reprises, dans 90 occurrences, les participants ont trouvé qu'il ne portait pas une charge émotionnelle assez conséquente. Ils ont décidé d'en multiplier l'usage pour insister et pour mettre davantage l'accent sur la grande charge émotionnelle de leur message (frustration, enthousiasme, joie, colère). [...] Les participants aux forums s'expriment avec un haut degré d'expressivité, et la transmission des émotions dans les forums est tout aussi importante que les messages eux-mêmes.

Murie s'interroge sur l'évolution de la ponctuation (ibid. : 151) :

Dans un monde virtuel où la sur-ponctuation est très fréquente, les signes utilisés de manière conventionnelle sont moins lourds de sens qu'ils ne le seraient dans d'autres domaines, par exemple dans un courrier formel où l'on n'utiliserait qu'un seul point d'exclamation pour exprimer sa surprise ou son exaspération.

Les contraintes à l'œuvre dans le cas de la communication médiatisée ont donc un effet sur la langue (ibid. : 255) :

Les contraintes de production et de réception des messages - utilisation d'un clavier, format du forum, différence d'espace-temps entre l'émetteur et le récepteur, 
production visible par tous les utilisateurs du forum, que le message soit destiné à l'ensemble des membres ou à un membre en particulier - ont un impact sur l' $\mathrm{ADF}^{1}$ en ce qui concerne le lexique utilisé, la ponctuation, les structures grammaticales et syntaxiques et l'orthographe.

À ces contraintes s'ajoutent, dans le cas qui nous préoccupe, celles de la situation d'apprentissage, apprentissage d'une langue, qui plus est collaboratif, donc l'obligation de compter sur ses pairs pour accomplir la tâche, de produire pour pouvoir être évalué (l'évaluation ne portant pas, dans le cas de notre corpus, sur l'exactitude syntaxique, mais sur la participation et la prise de risque langagière), la difficulté de devoir produire du langage sans en maîtriser tous les aspects. Ainsi, l'accumulation de points d'exclamation peut traduire une solution de facilité pour compenser une faiblesse langagière, mais peut être interprétée positivement comme une stratégie communicative.

En situation d'apprentissage, Phirangee et al (2016:73) notent en effet que les apprenants en ligne développent des stratégies et des conventions qui permettent à la communication médiatisée d'être aussi riche émotionnellement qu'en face-à-face, mais que cela peut être coûteux en temps et en effort. Les apprenants utilisent notamment les points d'exclamation de manière non conventionnelle (ibid. : 78). Pour ces auteurs, les étudiants en ligne écrivent sans correction immédiate, et utilisent les émoticônes ou points d'exclamation comme une stratégie pour inclure dans leur message des indices émotionnellement positifs pour minimiser le risque d'offenser quelqu'un inintentionnellement (ibid. : 83). House (2013 : 58) met en évidence que des locuteurs d'anglais langue étrangère entre eux réinterprètent, différemment d'un locuteur natif, les marqueurs de connectivité et d'intersubjectivité en fonction de leurs besoins de structuration du discours, au bénéfice de l'interaction avec l'interlocuteur. Herring (1999) note que si la cohésion est moindre dans la communication médiatisée (réponse séparée des questions, abandon de sujet de discussion, fils de discussions entremêlés ...), certains utilisateurs se saisissent de cette caractéristique pour en jouer dans une interactivité intensifiée.

Halvorsen (2012), qui étudie l'utilisation des émoticônes dans un forum d'apprentissage de l'anglais, remarque que des facteurs individuels entrent aussi en jeu, tels que la familiarité avec les forums et la familiarité avec les émoticônes. Carretero et al (2015) notent également le rôle de facteurs individuels. Dans leur étude d'interactions en ligne entre des étudiants autour de tâches pédagogiques, ils abordent l'exclamation à travers la typologie des actes expressifs (Searle, 1976) et remarquent une prédominance d'actes expressifs orientés vers les pairs plutôt qu'auto-centrés.

\footnotetext{
${ }^{1}$ Anglais des forums
} 


\section{MÉTHODOLOGIE}

\subsection{PRÉSENTATION DU CORPUS}

Les étudiants impliqués étudient les Sciences de l'éducation à distance dans le cadre d'un master 1. Ils suivent un module collaboratif de 8 semaines au cours desquelles ils pratiquent l'anglais et le numérique à travers un forum dédié de type Blackboard. Ils doivent construire de façon collaborative dans un Moo (environnement hypertextuel orienté objet) une institution éducative virtuelle, et en négocier les modalités sur le forum. Le corpus constitué est annoté mais n'est pas encore structuré en «corpus d'apprentissage » partageable tel que défini par Chanier et al. (2009:2) :

nous définissons la notion de corpus d'apprentissage en identifiant l'information qu'il doit contenir, structurée de façon à rendre possible son échange et la capitalisation des analyses. Le protocole de recherche, le scénario pédagogique, les interactions, productions et traces, les licences et les analyses capitalisables en sont les constituants.

L'absence de multimodalité dans notre corpus de productions écrites en rend le codage moins complexe qu'avec un corpus d'interactions par visio-conférence par exemple. Nous donnons quelques éléments quantitatifs ci-après de manière à situer les exemples sélectionnés pour l'analyse.

28 étudiants sont concernés par cette formation. 2039 messages au total sont publiés sur 8 semaines, dont 420 par la tutrice et 1619 par les étudiants. 24 étudiants utilisent des points d'exclamation, dans 442 messages sur la totalité (fig. 1).

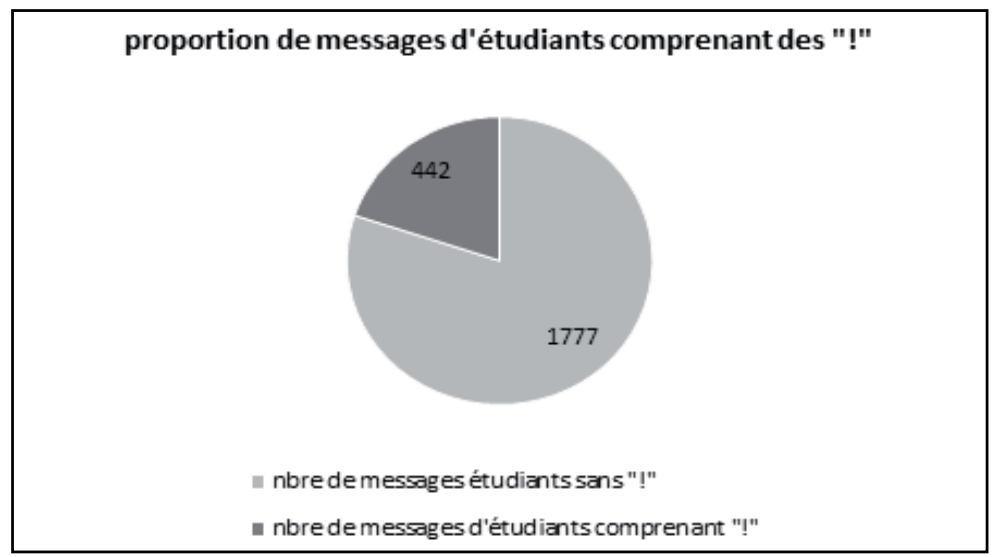

fig. 1 : proportion des messages comprenant un ou plusieurs points d'exclamation 


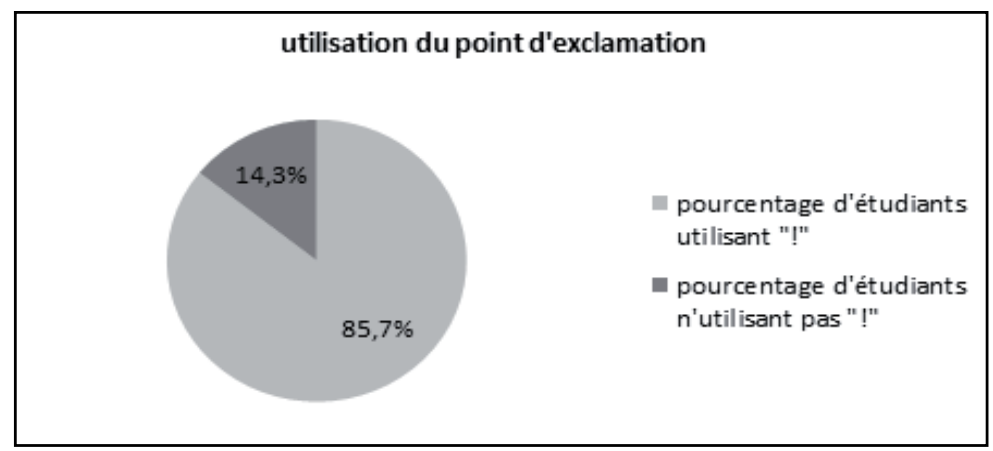

fig. 2 : pourcentage d'étudiants utilisant le point d'exclamation

Ainsi, une forte proportion d'étudiants utilise des points d'exclamation (fig. 2) mais une majorité de messages n'en contiennent pas. Il y a 1429 points d'exclamation au total dans les messages des étudiants, avec une moyenne de 3,2 points d'exclamation par messages (chaque message peut comporter plusieurs énoncés).

Le nombre de messages publiés, avec ou sans points d'exclamation, varie de 15 à 115 selon les étudiants, et le nombre de messages comprenant des points d'exclamation varie de 1 à 76 selon les étudiants. Le nombre de points d'exclamation utilisés va de 1 à 144 . On pourrait penser qu'un étudiant qui publie de nombreux messages est aussi un étudiant qui utilise beaucoup de points d'exclamation, mais ce n'est pas le cas. Il n'y a en effet pas de corrélation entre le nombre de messages et le nombre de points d'exclamation utilisés (fig. 3), en raison de variations individuelles telles que mentionnées en 2.6. Ainsi, nous ne pouvons représenter dans le même graphique l'étudiante 22 (fig. 4), en raison du nombre très élevé de points d'exclamation utilisés (466). Cette étudiante va jusqu'à utiliser 40 points d'exclamation dans un énoncé, comme le montre l'extrait suivant :

1) (etu22) 21 avril $201322: 45$ hello the girls, That's very great!!!! But I'm frustrated because there are few panels to fill. And I don't understand how to copy!!!! Help me!!!!!!!!!!!!!!!!!!!!!!!!!!!!!!!!!!!!!² A.

${ }^{2}$ Nous mettons en gras les segments pertinents pour l'analyse. 


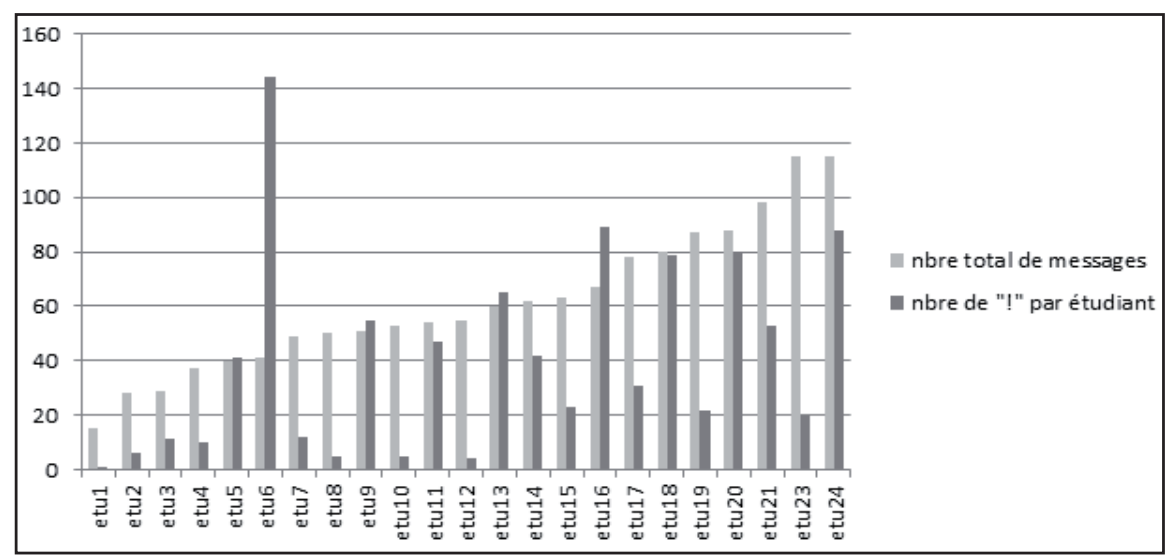

fig. 3 : nombre total de messages par étudiant et nombre de «!» par étudiant

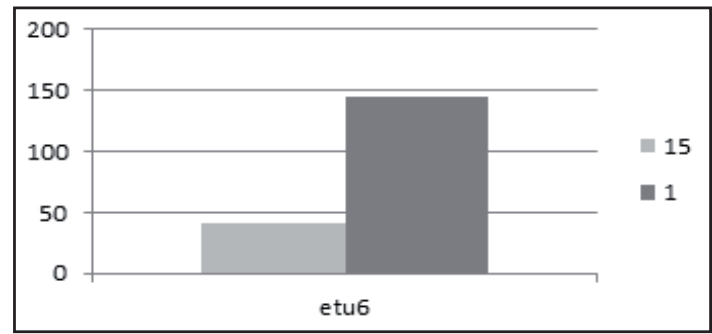

fig. 4 : nombre total de messages et nombre total de «!» pour l'étudiant 22

On pourrait penser également qu'un étudiant qui utilise les points d'exclamation dans une grande proportion de messages est forcément productif d'une manière générale, mais ce n'est pas le cas non plus (fig. 5). Il n'y a en effet pas de corrélation entre le taux de messages comprenant des points d'exclamation et le nombre total de messages.

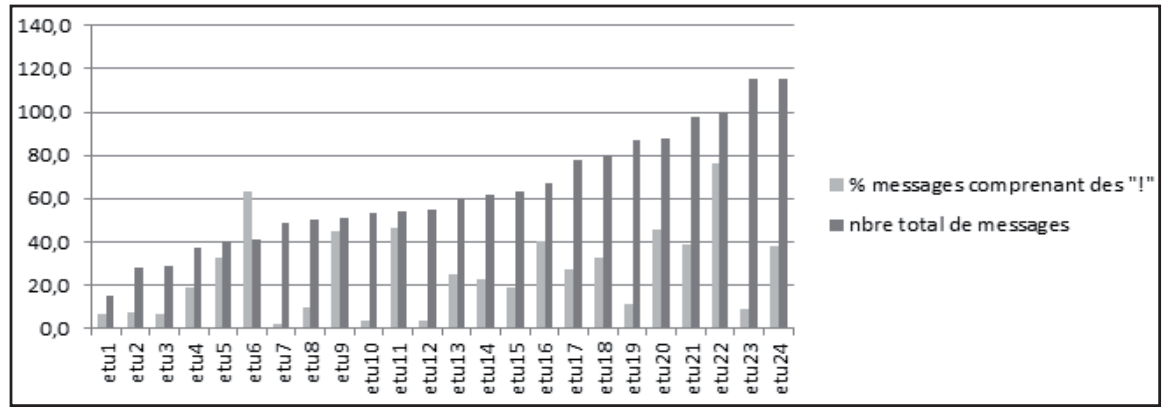

fig. 5 : nombre de messages par étudiant et taux de messages comprenant des «!» 
On pourrait imaginer enfin qu'un étudiant qui utilise les points d'exclamation dans une grande proportion de messages les utilise en grand nombre, mais ce n'est pas le cas non plus (fig. 6 et 7). Il n'y a en effet pas de corrélation entre le taux de messages comprenant des points d'exclamation et le nombre total de points d'exclamation.

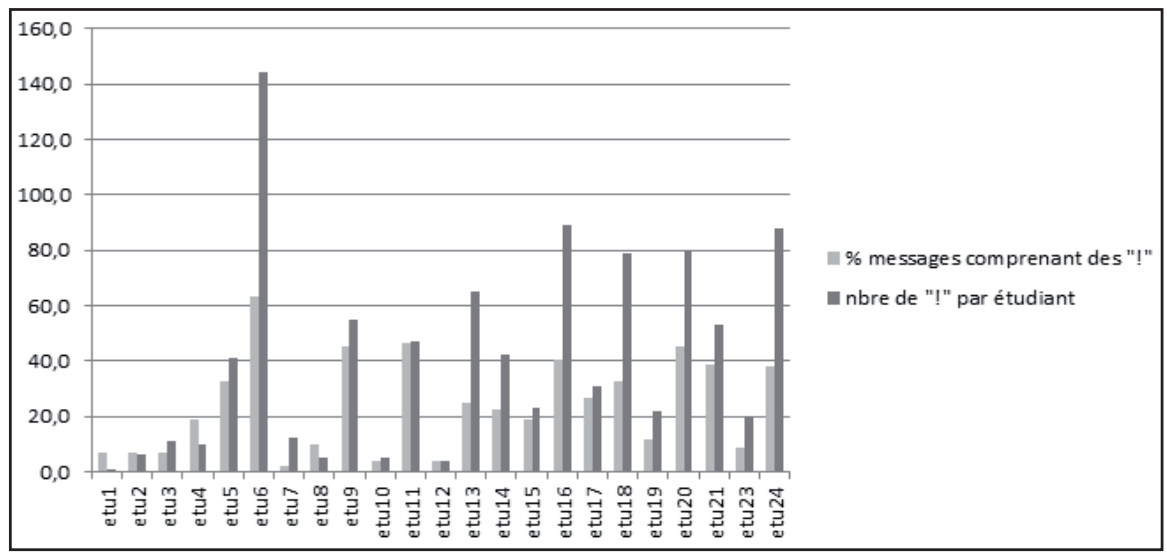

fig. 6 : taux de messages avec des «!» et nombre total de «!» par étudiant

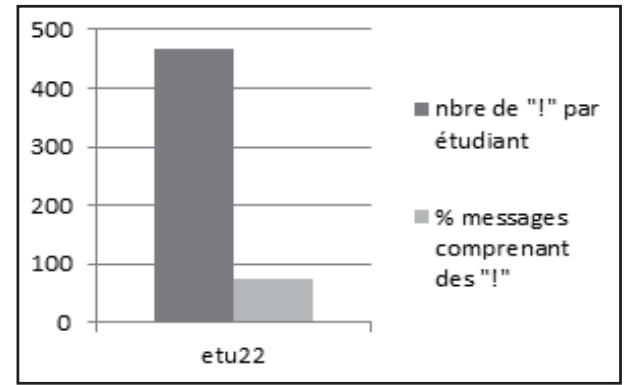

fig. 7 : taux de messages avec des «!» et nombre total de «!» pour l'étudiant 22

Après ces quelques données quantitatives, nous présentons les catégories utilisées pour l'annotation du corpus. Nous donnons également un éclairage quantitatif de ces catégories ci-dessous.

\subsection{MÉTHODE D'ANALYSE}

Dans notre corpus, plusieurs cas se présentent. Au niveau énonciatif, les points d'exclamation marquent l'intensité ou la référence à un préconstruit contraire. Au niveau pragmatique, ils marquent une relation avec la face des pairs ou du tuteur. Au niveau psychosocial, l'objectif est de faire avancer la tâche pédagogique tout en consolidant le lien social. La marque de l'intensité peut se combiner avec la référence au préconstruit ou avec la relation aux 
faces (Goffman, 1974) ou avec les deux simultanément. De même, la relation de préconstruction peut se combiner avec la marque de l'intensité ou la relation aux faces, ou les deux simultanément.

Nous avons donc annoté notre corpus avec les catégories suivantes, éventuellement combinées : intensité, préconstruit contraire, faces. Les différentes combinaisons apparaissant dans le corpus sont listées ci-dessous :

- intensité

- intensité/faces

- préconstruit

- préconstruit/faces

- intensité/préconstruit/faces

L'intensité se décline en intensité à valence positive ou négative. La relation aux faces se décline également en valence positive ou négative. Nous avons également annoté à part, sous l'étiquette «intensité phatique », les points d'exclamation contenus dans les énoncés d'ouverture et de fermeture. Les différentes combinaisons apparaissant dans le corpus, en tenant compte des valences, sont les suivantes, avec le comptage correspondant :

- intensité phatique : 150 (dont 26 dans un message au tuteur)

- intensité à valence positive : 19

- intensité à valence positive, en relation positive avec la face des pairs : 114

- intensité à valence positive, en relation positive avec la face du tuteur : 12

- intensité à valence négative, en relation négative avec la face du locuteur, donc positive avec la face des pairs : 32

- intensité à valence positive, en relation négative avec la face du locuteur (positive envers les pairs) : 1

- intensité à valence positive, en relation avec un préconstruit contraire : 55

- intensité à valence négative, en relation avec un préconstruit contraire : 10

- préconstruit contraire : 280 (dont 14 avec un notion d'accomplissement, exemple 9)(dont 22 dans des messages au tuteur)

- préconstruit contraire, en relation positive avec la face des pairs : 17

- préconstruit contraire, en relation positive avec la face du tuteur : 9

- préconstruit contraire, en relation négative avec la face du locuteur (positive envers les pairs) : 18 (dont 2 dans des messages au tuteur)

- intensité à valence positive, en référence à un préconstruit contraire, en relation positive avec la face des pairs : 2

- intensité à valence positive, en référence à un préconstruit contraire, en relation positive avec la face du tuteur : 3

- intensité à valence négative, en référence à un préconstruit contraire, en relation négative avec la face du locuteur (positive envers les pairs) : 2 
Tous ces aspects, quelle que soit la combinaison, vont dans le sens du renforcement de la qualité de la relation à l'autre en vue de la réalisation d'un travail commun (Quintin \& Masperi, 2010) et en vue du passage à un autre état du moi langagier par l'intermédiaire de ce travail commun. Cependant, certaines combinaisons semblent plus à même de marquer l'intersubjectivité que d'autres. Ainsi, il y aurait une gradation de l'intersubjectivité et un enchâssement des opérations énonciatives et socio-affectives autour de l'exclamation.

\section{ANALYSE D'EXEMPLES TIRÉS DU CORPUS}

\subsection{INTENSITÉ}

- intensité phatique : 150 messages (dont 26 dans un message au tuteur)

2) (etu10) 18 avril 2013 23:23 hello, if I understand, I must create anothr Room to bring up other picture ? I could integer after the other room in my first room ? thank you again!

Dans l'exemple 2, la formule «thank you again » cloture le message. Elle participe au renforcement de la qualité de la relation à l'autre. Les exemples de ce type, soit en ouverture, soit en fermeture, sont nombreux dans le corpus, comme on peut s'y attendre. On retrouve bien là la qualité conversationnelle de ces échanges mentionnée plus haut.

- intensité à valence positive : 19 messages

3) (etu6) 29 avril 2013 22:52 Good evening everybody! Back from my holidays in the south... F., I ' $m$ back home after a week in Marseille!!!!! Soooooo beautiful!!!! I will probably return there this summer.... Surely. So, it's OK for the debate on tuesday at 9pm. I'll be connected on the platform and I'll join the group for the conversation. To hear you tomorrow. Good night.

Dans l'exemple 3, nous présentons un exemple de haut degré avec la formule « Soooooo beautiful!!!! », où les 4 points d'exclamation s'accompagnent d'un allongement de la voyelle. Ici le haut degré ne semble pas accompagné d'une référence à une assertion contraire de manière évidente, ni être spécialement destinée à flatter la face des interlocuteurs. Il s'agit d'une mise en scène de l'intensité qui n'est pas spécifiquement tournée vers les pairs, même si, comme toutes les contributions sur le forum, elle a par définition un aspect conversationnel. A cet égard, l'analyse de Muris (2016 : 256) est éclairante :

l'individualité [...] semble être en opposition avec la notion de communauté, mais les deux existent en parallèle dans le corpus. En effet, les membres du forum, bien qu'ils essaient la plupart du temps de se conformer au reste du groupe, font aussi preuve de créativité pour exprimer leur individualité. Il est essentiel, pour comprendre les forums de discussion, de savoir que ces deux pôles n'entrent jamais en conflit, mais au contraire se nourrissent l'un de l'autre.

Ainsi, individualité et intersubjectivité ne sont pas incompatibles. 


\subsection{RÉFÉRENCE À UN PRÉCONSTRUIT / HYPERASSERTION}

- préconstruit contraire : 280 messages (dont 14 avec un notion d'accomplissement, exemple 9)(dont 22 dans des messages au tuteur)

4) (etu16) 4 avril 2013 23:04 Hello, i succeeded to create my account, me too ! A.

Dans l'exemple 4, l'étudiant asserte un accomplissement avec la formule signalée en gras. Là encore, il semble que l'individualité prenne le dessus. Les faces ne sont pas impliquées de manière évidente, et le haut degré, s'il est en arrière-plan, n'est pas la principale opération.

5) freedom 20 avril 2013 18:08 Yes, freedom for this week-end !!! I worked a lot today on the MOO so I prefer to eat sweets and look at a good movie !!!

L'exemple 5 présente également des hypersassertions, signalées en gras. L'assertion est affirmée contre toute attente. En effet, dans le contexte de ce master, il est improbable d'avoir un week-end de libre, et de pouvoir manger des bonbons en regardant un film. L'intersubjectivité est présente au sens ou le message contribue à la qualité de la relation à l'autre en partageant une assertion surprenante, mais elle ne semble pas centrale.

\subsection{INTENSITÉ ET PRÉCONSTRUIT}

Lors de la combinaison d'une opération impliquant un préconstruit contraire et l'expression de l'intensité, l'effet est là encore moins marqué d'intersubjectivité et plus tourné vers l'individualité.

- intensité à valence positive, en relation avec un préconstruit contraire : 55 messages

6) (etu9) 26 avril 2013 11:57 It's funny ! I like very much in the first video when the man erases the word with the tipex, it's unexpected ! I tested : - A hunter plays with a bear - A hunter speaks with a bear ( very funny the bear by the telephone) - A hunter kisses a bear ( with a little spray for the breath) - A hunter kills a bear ( it's the hunter the most frigntened !)

Dans l'exemple 6, en effet, l'hyperassertion face à l'inattendu (« it's unexpected »), est combinée ensuite avec le haut degré à travers l'adverbe «most » accompagné du point d'exclamation, au sujet d'un chasseur qui, contre toute attente, a peur d'un ours. L'intersubjectivité est présente dans l'aspect «partage d'information », mais l'implication des faces n'est pas centrale.

- intensité à valence négative, en relation avec un préconstruit contraire : 10 messages

7) (etu24) 29 avril 2013 17:07 Hi M., I can see that A. and you have connected your rooms to each of yours (except to the satellite) because now links appear in your rooms. But they don't appear in I.'s and mine and we can't create any connections for the moment...GGGRRRR!!! For the Hall, I think 
we could create it to doing more sense than a simply door but we don't really need this hall to connect our rooms... C.

L'exemple 7 est similaire au précédent, avec une intensité à valence négative, exprimée par l'interjection «GGGRRRR!!!! », qui combine majuscules, allongement et multiplication des points d'exclamation. Contre l'attente de l'étudiante, l'application informatique ne fonctionne pas. Là encore, l'intersubjectivité est présente dans le fait d'exprimer une frustration pour la partager avec les pairs, mais les faces ne sont pas impliquées outre mesure.

À la lumière d'une opposition entre production langagière sollicitée ou contrainte et une production non-sollicitée ou libre dans un cadre pédagogique, nous pouvons dire que ce qui relève de l'intensification est à la fois du domaine de l'investissement vers l'intersubjectivité et de l'investissement vers l'apprentissage de la langue. Barbéris (1995) estime, à propos des interjections, qu'elles «seraient donc des gestes vocaux : ambiguës et vagues comme les gestes, susceptibles de créer une synchronisation inconsciente entre les protagonistes de la communication, comme les gestes ». Certains s'interrogeront, dans la mesure où la solution des points d'exclamation est une solution économique du point de vue langagier pour l'apprenant, s'ils ne sont pas la trace d'un moindre effort. On pourrait alors arguer que l'intensité de l'effort langagier serait inversement proportionnelle à l'intensité intersubjective créée par les points d'exclamation lorsque les moyens linguistiques sont limités. Mais il faut rappeler que l'effort cognitif est déjà grand en soi, dans le contexte qui nous préoccupe, avec les contraintes de la distance, du numérique et de la langue étrangère s'additionnant. Nous pourrions donc voir au contraire ces points d'exclamation comme un engagement dans la tâche et un engagement dans l'échange langagier.

Signalons également, à propos de cette interjection, qu'elle est reprise par une autre étudiante dans d'autres messages avec un nombre de lettres et de points d'exclamation variable, manifestant ainsi une forme d'émulation. Les occurrences d'une forme doivent donc être interprétées à l'échelle du forum, voire à l'échelle de la sphère de communication toute entière de chaque participant. C'est ainsi que Herring (2013:16) parle de polychronicité, c'est-à-dire d'interactions multiples et simultanées à des rythmes différents. L'exemple 7 s'insère dans une séquence de quatre messages, deux avant (2 et 3 ) et un après (4), qui tous font état de difficultés techniques lors de la tâche en question.

Nous remarquons aussi que le contenu informationnel de l'exemple 7 cidessus est repris dans un autre message, le même jour, destiné au tuteur cette fois, qui ne présente ni interjection ni points d'exclamation.

8) (etu24) 29 avril 2013 16:43 Good afternoon J., I followed your instructions to connect my room [...] I tested all numbers, but I have always the same message... : (Thank you for help me 
La différence entre ces deux messages portant sur le même contenu informationnel nous permet de mettre en lumière le rôle de «GGGRRRR !!! » réservé à la construction de liance avec les pairs. Cette construction est d'ailleurs suivie d'effet, comme dans les exemples 9 et 10 où la frustration exprimée (« grr so i can’t see your robot !! ») trouve réponse dans le message d'une autre étudiante qui dit ne pas comprendre pourquoi elle ne voit pas le robot, et demande si parmi les pairs d'autres sont dans la même situation.

9) (etu 17) 19 mai 2013 15:03 [...] I don't see anything else...grr so i can't see your robot!! have a nice afternoon c.

10) (etu3) 19 mai 2013 21:55 Hello C., I don't understand why you can't see anything in Lab 2 ? My robot named Abby Sciuto is in the first room. Another person in the group can't see the second room of the laboratory ? Kiss F.

\subsection{INTENSITÉ ET PRÉCONSTRUIT : UN CAS PARTICULIER D'INTRASUBJECTIVITE}

Un cas particulier de combinaison de préconstruction et d'intensité apparaît lorsque les points d'exclamation renvoient à la remise en cause d'un univers de croyance (Martin Robert, 1987) qui concerne la représentation que l'étudiant a de son moi langagier. La langue pratiquée est une langue d'apprentissage et ce contexte implique une série de présupposés qui constituent cet « univers de croyance »: l'année de Master est difficile, apprendre l'anglais est difficile, le numérique est difficile, l'apprentissage à distance est difficile. Ainsi, une étudiante fait part d'une «petite satisfaction » («so, its a little satisfaction for me!!!!! ») après avoir écrit un résumé, ce dont elle ne se croyait pas capable.

11) 20 avril 2013 02:14 Hello J., i finished summary and i am very happy with my self and my dictionnary! i did'nt think to be capable to realize that!!!! so, its a little satisfaction for me!!!!! Good week-end. A.

Cette « petite satisfaction » devient une « vraie satisfaction » avec six points d'exclamation, quand, deux semaines plus tard, elle écrit un résumé d'un débat en ligne.

12) 4 mai $201323: 35$ job of week 5 Hello J., Here is the summary of our debate! This is easier than using the tool because I had all my time alone with my friend the dictionary!!!!! But, I feel I have made great strides in a month!!! A true satisfaction!!!!!! A.

Il y a d'autres exemples similaires dans le corpus, qui, en même temps qu'ils contribuent à l'intensification globale de l'intersubjectivité dans le forum, signalent une relation intrasubjective avec un état antérieur du moi apprenant de langue étrangère. L'intersubjectivité n'est donc pas centrale ici. Il s'agit plutôt d'une contribution orientée vers l'individualité. D'ailleurs, les pairs ne réagissent pas à ces commentaires, qui participent quand-même d'une dyna- 
mique générale de reliance sur le forum en mettant en relief des difficultés et en mettant en scène un cheminement intime en le dévoilant pour les pairs.

\subsection{INTENSITÉ ET FACES}

Avec la combinaison de l'expression de l'intensité et de l'implication des faces, l'intersubjectivité apparaît plus pleinement.

- intensité à valence positive, en relation positive avec la face des pairs : 114 messages

13) (etu9) 22 avril 2013 11:41 Hello V. and the girls Very good idea the creation of the forest ! It sticks well on the philosophy of our institution. The children experiment, are in phase of discovery... Of more the natural side of this space is interesting as well as the raising awareness to the environmental causes! bye $\mathrm{J}$.

Dans l'exemple 13, la face de l'interlocuteur est flattée avec la formule indiquée en gras. De plus, cette relation est intensifiée avec l'adverbe « very ».

- intensité à valence positive, en relation positive avec la face du tuteur : 12 messages

14) (etu9) 1 mai 2013 10:38 Thank your for this list J. ! there are topics which inspire me a lot !

Il en est de même avec l'exemple 14, cette fois destiné au tuteur. Cette fois, c'est l'adverbe « a lot » qui aacompagne le point d'exclamation.

- intensité à valence négative, en relation négative avec la face du locuteur, donc positive avec la face des pairs : 32 messages

15) (etu22) 25 avril 2013 22:16 Yes C., For me, I think I 'don't have a very english for understand this game!!! A.

La face de l'interlocuteur est parfois flattée indirectement, comme dans l'exemple 15. En effet, c'est en mettant en scène ses difficultés avec l'adverbe « very » et les 3 points d'exclamation que le locuteur se met en position d'être éventuellement conseillé par ses pairs, ou met les pairs en position de se sentir valorisés car ils n'ont pas les mêmes difficultés.

- intensité à valence positive, en relation négative avec la face du locuteur (positive envers les pairs) : 1 message

16) (etu15) 20 avril 2013 22:25 I killed 26 personns and injuried 26 more... But no damages to the buildings... What a success :

L'exemple 16 présente une opération plus complexe, ce qui explique qu'elle n'apparaisse qu'une fois dans le corpus. L'étudiant pratique l'autodérision et l'ironie en utilisant l'intensité positive du mot interrogatif « what » accompagné du point d'exclamation, pour un effet négatif sur sa propre face, de manière à valoriser les pairs indirectement. 


\subsection{PRÉCONSTRUIT ET FACES}

Avec la combinaison d'une hyperassertion et d'une relation aux faces, là encore, l'intersubjectivité est plus centrale.

- préconstruit contraire, en relation positive avec la face des pairs : 17 messages

17) (etu17) 3 avril 2013 21:06 hi E., nice to talk to you too. I saw your message on an other forum.. I hope you 'Il find a group and work on this TC4 serenely! we are all tired now, don't give up!! see you later

Dans l'exemple 17, le locuteur-apprenant met en scène une assertion allant à l'encontre de la possibilité de ne pas trouver de groupe ou d'abandonner la formation destinée à un pair, tour à tour sous forme d'une projection, puis sous la forme d'une injonction.

- préconstruit contraire, en relation positive avec la face du tuteur : 9 messages

18) (etu18) 6 mai 2013 19:47 Hello J., I spend you two creations made with WORDLE. The first concerns a blog and the second my TER. It a funny tool !!! I'm going to test the second tool. Thank you for sharing it with us ;-)

Avec l'exemple 18, c'est la face du tuteur qui est flattée avec l'assertion que l'outil proposé par le tuteur est, contre toute attente, un outil amusant ( a funny tool »).

- préconstruit contraire, en relation négative avec la face du locuteur (positive envers les pairs) : 18 messages (dont 2 dans des messages au tuteur)

19) (etu9) 25 avril 2013 11:40 Hello the girls Here i am again ! ( I write you of the bottom of my bed ! ) always with this bad flu nevertheless we are for spring but germs are resistant this year! But i feel a little better as even. I am thus going to create the playing field in Moo as expected.

La même opération peut être mise en place, cette fois de manière indirecte, avec une mise en scène négative de la face du locuteur, combinée avec une hyperassertion : il est improbale qu'un apprenant travaille tout en étant alité. Cette mise en relief d'une faiblesse du locuteur valorise par un effet miroir la face des pairs.

\subsection{INTENSITÉ, PRÉCONSTRUIT ET FACES}

Peu d'exemples combinent l'expression de l'intensité, l'hyperassretion et l'implication des faces. Ceci n'est pas surprenant, puisque les apprenants n'ont pas forcément tous les outils langagiers pour effectuer cette opération relativement complexe. Avec ces exemples, l'intersubjectivité est centrale.

- intensité à valence positive, en référence à un préconstruit contraire, en relation positive avec la face des pairs : 2 messages 
20) 28 mai 2013 10:24 Hello the girls It's the end of our adventure TC4 ! It was a pleasure to work with you, our team worked well and i think that we made a good job! Good luck in all for the continuation and for the revisions ! it's our final straight ! Good day J.

Dans l'exemple 20, le locuteur fait référence au préconstruit selon lequel la formation est longue et difficile. Contre toute attente, elle est proche de sa fin. L'adjectif « final » participe à l'expression d'un intensité, et le pronom « our », par lequel le locuteur s'inclut dans l'énoncé, renforce l'intersubjectivité. L'étudiante encourage ses pairs tout en se montrant solidaire.

- intensité à valence positive, en référence à un préconstruit contraire, en relation positive avec la face du tuteur : 3 messages

21) 27 mai 2013 10:48 Hello J. I appreciated this TC. I discovered a new way of working very interesting and rich in discoveries ! I estimated a lot all dicoveries ( test games, discover new computing tools..) and to speak English permanently allows to improve in the language! This TC generated many exchanges and $\mathrm{i}$ find it pleasant because i like communication and I find it very well to communicate so. The work on Moo was very pleasant too, after some difficulties at the beginning to get acquainted with this one, then it really was a pleasure to create these rooms and to imagine our institution. Finally we benefited from a good supervision with a regular followup from you what is encouraging and tempts to put a lot ! Thank you J. J. ps : Many exchanges 4067 ! It's good it's the platform which " lives “"

Les opérations sont combinées dans l'exemple 21 , là encore de manière complexe et orientée vers l'intersubjectivité. En effet, contre toute attente, l'étudiante a « découvert une nouvelle manière de travailler », qu'elle qualifie à l'aide de l'adjectif « interesting », accompagné de l'adverbe « very » et d'un point d'exclamation. Le nom « discoveries » et le verbe « discovered » intensifient encore l'idée d'inattendu. Le fait de partager ces remarques avec le tuteur valorise celui-ci, puisqu'il a mis en place le scénario pédagogique.

- intensité à valence négative, en référence à un préconstruit contraire, en relation négative avec la face du locuteur (positive envers les pairs) : 2 messages

22) (etu22) 7 avril 2013 00:05 Hello girls, for to follow on our brainkwork, i have to try to regroup the ideas of group. I put the synthésis in our mails. I hope to have respect our ideas!!!!!!! Tell to me and excuse me for my little inglish hihihihi!!!!Kiss A.

La complexité est encore augmentée lorsque la valorisation des interlocuteurs se fait de manière indirecte par la mise en scène des difficultés du locuteur. Ici, l'étudiante rit d'elle-même « hihihihi!!!! » et intensifie cette autodérision avec la multiplication des points d'exclamation et l'allongement de l'interjection. Elle qualifie son anglais de « little». Elle fait référence au préconstruit selon lequel un certain niveau d'anglais est requis pour participer. 
Nous avons vu que selon les combinaisons d'opérations énonciatives présentes, l'intersubjectivité peut être centrale ou au contraire sous-jacente. Seule l'intensité phatique est intersubjective sans être combinée à d'autres opérations.

\subsection{ESPACE D'INTENSITÉ INTERSUBJECTIVE}

Dans certains cas, le point d'exclamation semble signaler un véritable espace intersubjectif sur le forum, une parenthèse intimiste de forte intensité intersubjective. En début de module, la tutrice poste un message évoquant la sphère personnelle en signalant qu'elle va au lit, contre toute attente, ce préconstruit contraire concernant autant le fait d'aller au lit que le fait de le signaler sur le forum.

\section{3) 3 avril 2013 22:23 BED I'm going to bed !!}

Ce message génère une suite de contributions gratuites orientée entièrement vers la reliance et non plus à propos de l'avancement des tâches pédagogiques. Etu3 par exemple, joue sur les mots en calquant le «yes we can» d'Obama avec un «yes we do!!» en hyperassertant la capacité d'elle-même et de ses pairs à compléter la formation. La combinaison [hyperassertion/implication positive de la face des pairs] est bien présente.

24) (etu3) 9 avril 2013 22:40 It would be necessary to believe the theory according to which we learn by sleeping for not discouraged. yes we do !!

Si ce n'est pas le point d'exclamation en soi qui est à l'origine des contributions suivantes, on voit comment il peut être au cœur d'un processus de tissage intersubjectif. Dans une autre rubrique du forum, cette émulation intersubjective semble se produire de nouveau de manière similaire. La tutrice poste un message humoristique indiquant que la plate-forme implosera si les 2000 messages sont atteints.

25) 21 mai 2013 21:23 FORSE will implode if we reach 2000 messages ;-)

Une étudiante relève le défi et fait une allusion humoristique à une combinaison de nuits courtes et café, avec une liste qui indique l'intensité par accumulation («Perseverance, motivation, concept, short nights and coffee ») et accumulation de points d'exclamation également. Elle qualifie de familiale cette aventure pédagogique, contrairement à ce qu'on pourrait croire dans l'univers de croyance partagé, dans lequel le master est un diplôme universitaire qui n'implique pas la famille. La combinaison maximale [hyperassertion/implication positive de la face des pairs/intensification] est illustrée dans cet exemple.

26) (etu23) 23 mai 2013 22:31 It is on! Perseverance, motivation, concept, short nights and coffee!!!!!!!It's hard but what a pleasure to go further. A true family adventure!! A.

Un autre espace d'intensité intersubjective semble apparaître avec une rubrique créée par la tutrice, consacrée aux compliments, et caractérisée de 
nouveau par la présence de nombreux points d'exclamation. La tutrice y poste un message pour célébrer le fait qu'une étudiante ayant eu de gros problèmes de connexion arrive enfin à se connecter à la plate-forme numérique, contre toute attente.

27) 19 avril 2013 10:52 let's celebrate !!!! D. can MOOOOOOOOO ! from the cybercafe... it's better than nothing!!

Ce message déclenche, ou tout du moins est suivi de 18 messages qui comprennent tous soit des points d'exclamation, soit de l'humour, soit des références lexicales à la collaboration et à l'encouragement. L'échange suivant par exemple, s'étend sur 5 messages filés qui suivent le thème de la « Guerre des étoiles». Le premier message remercie pour des félicitations (intensité phatique) et cite maître Yoda, ce qui entraîne une réponse humoristique de etu6 qui demande si Yoda est son père. Etu22 répond en hyperassertant avec quatre points d'exclamation que etu23 est la fille de Yoda. Etu23 continue sur le thème en disant que Leila est sa sœur, Luke son frère et contre toute attente ses ciseaux à ongle un sabre laser. Une autre étudiante, qui n'est pas intervenue dans l'échange jusque là, conclut avec le slogan « que la force soit avec toi !!! », avec le préconstruit contraire qu'un étudiant pourrait avoir des difficultés, les faces étant donc impliquées avec une valence positive.

28) (etu23) 20 avril 2013 13:26 thank you for your congratulation !! as Maitre Yoda said me yesterday : "May the Force be with us" I.

29) (etu6) 20 avril 2013 17:52 Is Yoda your father?....

30) (etu22) 20 avril 2013 18:23 YES, n.! I. is a Yoda's girl!!!! As you see i., you can see the ressemblance ,no? It's all in the attitude....!!!! Kiss A.

31) (etu23) 20 avril 2013 18:46 Yes my friends... Leila is my sister, and luke is my brother in law... my nail clippers is a lazer sword ! I will show you in june. I.

32) (etu12) 21 avril 2013 19:36 That the strength is with you!!! v.

Si ce ne sont pas les points d'exclamation sur lesquels reposent tout l'échange, ils sont néanmoins impliqués étroitement avec l'humour et l'intensification intersubjective à l'œuvre.

\section{CONCLUSION : APPROCHE ÉNONCIATIVE ET ASPECTS PSYCHOSOCIAUX}

À travers l'étude des points d'exclamation dans les productions écrites d'apprenants de langue sur un forum d'apprentissage à distance, nous avons souhaité apporter un éclairage sur les aspects intersubjectifs de l'exclamation. Nous avons rappelé le caractère intrinsèquement intersubjectif de l'énonciation elle-même, puis mis en relation cet aspect avec les caractéristiques de la communication médiatisée, avec le fonctionnement d'un forum collaboratif et les états intrasubjectifs successifs de l'apprenant de langue. Nous avons vu que le 
point d'exclamation doit parfois être interprété à l'aune d'une rubrique entière du forum ou tout du moins en regard de la dynamique d'un échange long. Le point d'exclamation apparaît dans ce contexte comme un marqueur d'intensité intersubjective. A ce titre, si l'on souhaite, à l'inverse, provoquer cette intensité que l'on estimerait bénéfique du point de vue de l'apprentissage, on s'attacherait alors à créer cet espace pour laisser toute la place à cette intersubjectivité.

Nous avons vu qu'au-delà d'opérations analysées de manière discrète telles que le marquage du haut degré ou de l'hyperassertion, c'est dans la combinaison des opérations qu'il faut chercher l'interprétation des points d'exclamation sur un forum d'apprentissage. Cette combinaison permet d'envisager une gradation de l'intersubjectivité qui nous amène vers la conciliation de l'énonciatif et du psychosocial ou socioaffectif, de l'individuel et du transindividuel, de l'interpersonnel et de l'interlocutif.

Nous souhaitons que cette approche contribue à apporter un éclairage sur l'exclamation en général du point de vue des relations intersubjectives qu'elle peut construire.

\section{BIBLIOGRAPHIE}

Angermuller, J. (2014), Poststructuralist Discourse Analysis: Subjectivity in Enunciative Pragmatics, Palgrave Macmillan.

Anis, J. (1998), Texte et ordinateur : l'écriture réinventée ? Bruxelles : De Boeck.

Anis, J. (1994), « Pour une graphématique des usages : le cas de la ponctuation dans le dialogue télématique », LINX, 31, 81-97.

Bakhtine, M. ([1952-53], [1979], 1984), « Les genres du discours », in Esthétique de la création verbale, Paris : Seuil, 263-308.

Barbéris, J.-M. (1995), «L'interjection : de l'affect à la parade, et retour », Faits de langues, 6, L'exclamation, 93-104.

Benveniste, E. (1974), Problèmes de linguistique générale II, Paris : Gallimard.

Bouscaren, J. et Chuquet, J. (1987), Grammaire et textes anglais, Guide pour l'analyse linguistique, Paris : Ophrys.

Bres J. (2012), «Énonciation et dialogisme : un couple improbable ? », in Dufaye L. et Gournay L., (éd.), Benveniste après un demi-siècle. Regards sur l'énonciation aujourd'hui, Paris : Ophrys, 3-24

Carretero, M., Maíz-Arévalo, C. et Angeles Martínez M. (2015), « An analysis of expressive speech acts in online task-oriented interaction by university students », Procedia - Social and Behavioral Sciences, 173, 186-190. 
Chanier T., Ciekanski M. (2009). «Du partage des corpus à l'analyse des interactions en ligne dans des situations d'apprentissage : quelle méthodologie pour la recherche sur corpus d'apprentissage ? », in Develotte, Mangenot, Nissen (coord., 2009) Actes du colloque Echanger pour apprendre en ligne (EPAL). Grenoble, 5-7 juin 2009. Consulté le 12/01/2017. URL : http://epal.u-grenoble3.fr/actes/

Crystal, D. (2001), Language and the Internet, Cambridge : Cambridge University Press.

Culioli, A. (1973), « Sur quelques contradictions en linguistique », Communications, 20, 83-91.

Culioli, A. (1999), Pour une linguistique de l'Énonciation - Formalisation et opérations et repérages (Tome 2), Paris : Ophrys.

Culioli, A. (2002), Variations sur la linguistique, Paris : Klincksieck.

Develotte, C. et Codreanu, T. (2010), « Enseigner par visioconférence poste à poste : cadre méthodologique pour l'analyse de pratiques tutorales », in Sidir, M., Bruillard, E., Baron, G.-L., Acteurs et objets communiquants. Vers une éducation orientée objets ? Journées Communication et Apprentissages Instrumentés en Réseau 2010, Lyon, INRP, p. 60-71. http://halshs.archives-ouvertes.fr/hal-00510826/

Devilla, L. (2006), « Analyse de La linguistique textuelle -Introduction à l'analyse textuelle des discours », Alsic [En ligne], 9, document alsic_v09_14-liv4, mis en ligne le 1er Décembre 2006, consulté le 25 Mars 2014. URL: http://alsic.revues.org/300

Douay C. (2003), « Des modalités de l'interlocution au système des modaux », Corela [En ligne], 1(1), mis en ligne le 01 avril 2003, consulté le 09 mai 2016. URL : http://corela.revues.org/636

Filippi-Deswelle, C. (2013), « Du locuteur au sujet énonciateur-locuteur, Théorisation des métatermes 'locuteur/allocutaire' en 'énonciateur/coénonciateur' : de la 'mécanique interlocutoire' de Benveniste au couple énonciatif de Culioli », in Benveniste après un demi-siècle, Regards sur l'énonciation aujourd'hui, Lionel Dufaye et Lucie Gournay (éds.), Paris : Ophrys, 84-108.

Filippi-Deswelle, C. (2010), « Quand “je” est un "autre” ou quand though, marqueur de relance discursive (d'“afterthought"), introduit une altérité intrasubjective », in Dufaye L. et Gournay L. (éds), L'Altérité dans les théories de l'énonciation, collection Langues, Langage et Textes, Paris : Ophrys, 37-55.

Gélis, J.-M. (2013), « L'engagement des enseignants dans un dispositif d'enseignement à distance », Distances et médiations des savoirs [En ligne], 2 , mis en ligne le 18 février 2013, consulté le 09 mai 2016. URL : http://dms.revues.org/175

Goffman, E. (1974), Les rites d'interaction, Paris : Minuit. 
Guichon, N. (2012), «L〉apprentissage des langues médiatisé par les technologies (ALMT) - Étude d> un domaine de recherche émergent à travers les publications de la revue Alsic », Alsic [En ligne], 15(3), mis en ligne le 15 décembre 2012, consulté le 04 janvier 2017. URL : http://alsic.revues.org/2539

Guillaume, B. et Baumer, E. (2014), «Expression de l'exclamation en anglais au moyen de marqueurs atypiques », Corela [En ligne], 12(2), mis en ligne le 15 décembre 2014, consulté le 10 mai 2016. URL : http://corela.revues.org/3654

Halvorsen, A. (2012), « Patterns of Emoticon Usage in ESL Students' Discussion Forum Writing », CALICO Journal, 29(4), 694-717.

Henning, N. (2013), « La polyphonie et la ScaPoLine 2011: l'héritage benvenistien, nouvelles perspectives, avancées théoriques et épistémologiques », in Benveniste après un demi-siècle, Regards sur l'énonciation aujourd'hui, Lionel Dufaye et Lucie Gournay (éds.), Paris : Ophrys, 129-153.

Herring, S. C. (1999), «Interactional coherence in CMC », Journal of Computer-Mediated Communication, 4 (4), 1-13.

Herring, S. C., Stein, D., et Virtanen, T. (eds.) (2013), « Introduction to the pragmatics of computer-mediated communication », Handbook of pragmatics of computermediated communication, 3-31. Berlin: Mouton.

House, J. (2013), « Developing pragmatic competence in English as a lingua franca: Using discourse markers to express (inter)subjectivity and connectivity », Journal of Pragmatics, 59(A), 57-67.

Jeanneau, C. et Ollivier, C. (2009): «Eléments influençant la nature des interactions en ligne des apprenants de langues », in Develotte, Mangenot, Nissen (coord.) Actes du colloque Echanger pour apprendre en ligne (EPAL). Grenoble, 5-7 juin 2009.

Kerbrat-Orecchoni, C.(1980), L'Énonciation : de la subjectivité dans le langage, Paris : A. Colin.

Martin, R. (1987), Langage et croyance : les «univers de croyance» dans la théorie sémantique. Liège : $\mathrm{P}$. Mardaga.

Mellet, S. (2011), « Lionel Dufaye, Lucie Gournay - L'altérité dans les théories de l'énonciation. Paris / Gap : Ophrys (collection « Langues, langage et textes »), 2010, 194 pages, $20 € »$, Corpus [En ligne], $10 \mid 2011$, mis en ligne le 18 juin 2012, consulté le 09 mai 2016. URL : http://corpus.revues.org/2143

Mosès, S. (2001), «Émile Benveniste et la linguistique du dialogue. », Revue de métaphysique et de morale 4/2001, 32, 509-525. URL : www.cairn.info/revue-de-metaphysique-et-de-morale-2001-4-page-509.htm. DOI : 10.3917/rmm.014.0509.

Muller, C. (2016), «Les relations interpersonnelles, un facteur prédominant dans les biographies langagières », in Voise A.-M. et Collière C., Apprendre de l'intime : Entre littérature et langues, EME éditions : Louvain-la-Neuve, 287-305. 
Murie, C. (2016), L'anglais des forums : caractéristiques lexicales, syntaxiques et stylistiques. Thèse soutenue le 5 décembre 2016, sous la direction de Albrespit J., Université Bordeaux Montaigne.

Nølke, H. (2013), «La polyphonie et la ScaPoLine 2011 : l'héritage benvenistien, nouvelles perspectives, avancées théoriques et épistémologiques», in Dufaye L. et Gournay L., (éd.), Benveniste après un demi-siècle. Regards sur l'énonciation aujourd'hui, Paris : Ophrys 129-153.

Paillard, D. (2013), « Les marqueurs discursifs comme catégorie », in Dufaye L. et Gournay L., (éd.), Benveniste après un demi-siècle. Regards sur l'énonciation aujourd'hui, Paris : Ophrys, 154-181.

Paveau, M.-A. (2013), « Technodiscursivités natives sur Twitter. Une écologie du discours numérique », dans Liénard, F. (2013), (éd.), Culture, identity and digital writing, Epistémè, 9, Revue internationale de sciences humaines et sociales appliquées, Séoul : Université Korea, Center for Applied Cultural Studies, 139-176.

Phirangee, K. et Hewitt, J. (2016), « Loving This Dialogue!!!: Expressing Emotion Through the Strategic Manipulation of Limited Nonverbal Cues in Online Learning Environments », in Tettegah L. et McCreery M. (éd.), Emotions, Technology, and Learning, 1. New York: Elsevier.

Polge-Loï, V. (2013), « Interculturalité où es-tu? Réflexion sur l'intercompréhension en situation d'échanges exolingues en classe de langue », in Actes du Colloque EPAL 2013 (Echanger pour apprendre en ligne), Dejean-Thircuir, Charlotte, Mangenot, François, Nissen, E. et Soubrié, T. (2013) (éd.), Université Grenoble Alpes, 6-8 juin 2013.

Searle, J. R. (1976), « A classification of illocutionary acts », Language in Society, 5(1), 1-23.

Torres i Vilatarsana, M. (2001). « L`analyse du discours médiatisé par ordinateur : 1>apport de la linguistique à la société de l>information », in Actes du Colloque «La Communication Médiatisée par Ordinateur : un carrefour de problématiques ». Université de Sherbrooke, 15-16 mai.

Von Münchov, P. et Rakotoelina, F. (2006). « L'interrogation et le discours rapporté dans les forums de discussion sur l'environnement en français et en anglo-américain ». Les Carnets du CEDISCOR, 9, 93-112.

Yun, H. (2009), « Échanges à distance entre apprenants de FLE. Etude des interactions synchrones en contexte académique », Thèse de doctorat, Université de Sorbonne Nouvelle : Paris 3.

Zourou, K. (2006), « Apprentissages collectifs médiatisés et didactique des langues : instrumentation, dispositifs et accompagnement pédagogique », Thèse de doctorat, Université Stendhal : Grenoble III. 